\title{
Erste Ergebnisse neuerer Untersuchungen des Klettgauer Lößes
}

\section{Peter Fitze}

\section{Problemstellung und Untersuchungsgebiet}

Methodische Verfeinerungen sowie das immer zahlreicher werdende Material auf dem Gebiet der Bodenkunde und der Lößstratigraphie enthüllen immer neue Details über Klima und Landschaft des Quartärs, wenn dies auch häufig nur für die letzte Kaltzeit und auch dann nur für bestimmte Lokalitäten gilt. In der Schweiz beschränkt sich dieser Raum hauptsächlich auf die Periglazialgebiete der Würmkaltzeit, zu denen auch das Klettgau zu rechnen ist.

Innerhalb solcher Periglazialgebiete ist als besondere Schwierigkeit mit einer Vielfalt von formbil- denden Prozessen auf kleinem Raum zu rechnen: Erosion und Akkumulation durch Schmelzwasserflüsse, Windakkumulation von Löß und Sand, Denudation durch solifluidale Vorgänge (z. T. wieder mit großflächiger Akkumulation verbunden) sowie die typischen Prozesse, die sich in und über dem Dauerfrostboden abspielen.

Der Arbeitsraum, in dem ich lößstratigraphische Untersuchungen zu machen beabsichtigte, ist aus Fig. 1 ersichtlich. Grundsätzlich läßt sich aus dieser Skizze herauslesen, da $\beta$ das Gebiet WilchingenHallau-Neunkirch aus verschiedenen Lößflächen besteht, die durch Niederterrassenschotter voneinander getrennt werden (oft auch durch Rißschot-

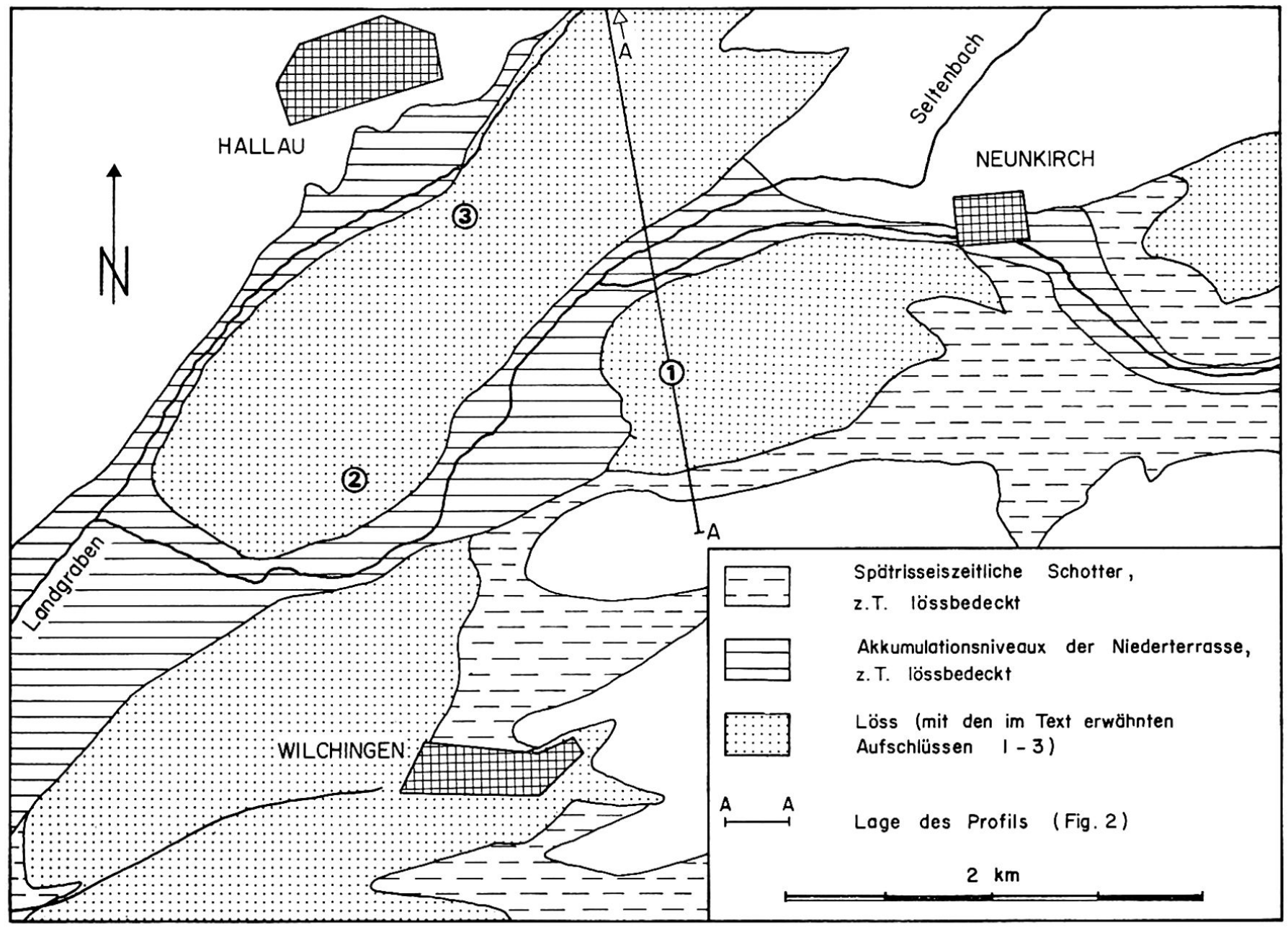

Figur 1. Geologische Skizze des Untersuchungsgebietes (nach der geologischen Karte von Hantke, 1967) 
ter). Die vorläufigen Untersuchungsergebnisse, gewonnen an einigen Aufschlüssen innerhalb dieser Lößgebiete, sollen in dieser kleinen Arbeit zur Sprache kommen. Genauere Abklärungen, auch im weiteren Gebiet des Klettgau, fehlen aber noch und sollen in nächster Zeit gemacht werden. Wenn auch die Ergebnisse noch nicht als endgültig betrachtet werden dürfen, schienen sie mir zur Veröffentlichung doch wichtig genug, da sie eindeutig nach einer Korrektur unserer Vorstellung von den Verbreitungsgebieten des Lößes rufen.

\section{Ergebnisse der Untersuchungen an den Aufschlüssen}

Die Mehrzahl der auf älteren Karten eingezeichneten Gruben ist heute leider zugeschüittet. Dies trifft besonders in den mit «Löß» bezeichneten Gebieten zu. Eine Hilfe bildet aber der Untersuchungsbericht über die Grundwasserverhältnisse im Klettgau (EAWAG, 1969) mit verschiedenen Bohrungen und Baggerschlitzen. Dieser Bericht wurde mir freundlicherweise vom Kantonsingenieur des Kantons Schaffhausen zur Verfügung gestellt.

Bei der Begehung des Gebietes zeigte sich rasch, daß die Grube auf dem "Hungerbuck» (Nr. 1 in Fig. 1) die interessantesten Aspekte zeigte, weshalb sich das Hauptaugenmerk auch auf diesen Aufschluß richtete. Vorerst aber sollen die beiden andern Aufschlüsse (Nr. 2 und Nr. 3 in Fig. 1) kurz besprochen werden.

\subsection{Kiesgrube Nr. 2}

An der Nordwestwand dieser Grube, in der Nähe der Station Wilchingen-Hallau, ergab sich an einer Stelle (Pt. 676 630/281 500; 415 m ü. M.) folgender Aufbau der Deckschicht, die die mächtigen rißzeitlichen (?) Schottermassen überlagert: $\mathrm{Zu}$ oberst liegt lehmiges Material von $1 \mathrm{~m}$ Mächtigkeit, darunter folgt ein ebenfalls etwa $1 \mathrm{~m}$ mächtiger lehmiger Sand. Den Abschluß gegen die Schotter bilden $80 \mathrm{~cm}$ eines stark tonigen Materials. Vereinzelt wurden in den obersten $2 \mathrm{~m}$ gerundete Kieselsteine von Haselnußgröße gefunden. Im lehmigen Sand ist ein schöner $B_{t}$ (= Tonanreicherungshorizont) einer Bänderparabraunerde ausgebildet (Abb. 1). Das ganze Profil kann aber nicht als Parabraunerde angesprochen werden, da ein $\mathrm{A}_{1}$-Horizont (= Tonauswaschungshorizont), der über dem $\mathrm{B}_{\mathrm{t}}$ liegen sollte, nicht nachgewiesen werden kann. Schwierig ist es, diesen $B_{t}$ altersmäßig einzustufen, denn das Alter des Schotters im Liegenden dieses Bodens kann nicht mit Sicherheit als rißeiszeitlich angenommen werden.

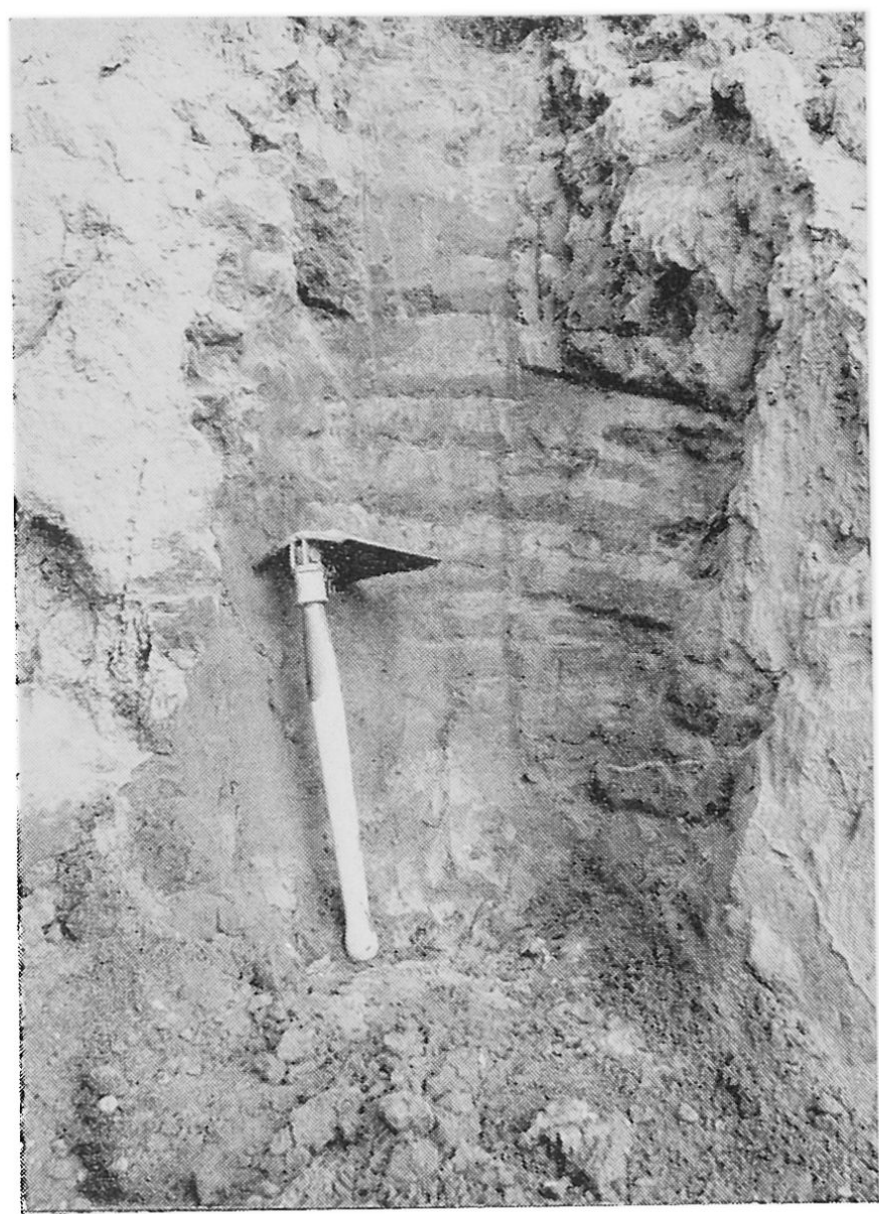

Abbildung 1. Gutausgebildeter $\mathrm{B}_{\mathrm{t}}$-Horizont der im Text erwähnten Bänderparabraunerde von fast einem Meter Mächtigkeit (Pt. 676 630/281 500)

\subsection{Kiesgrube Nr.3}

Auf der Nordwestseite dieses Aufschlusses (bei Pt. 677 150/282 780; $417 \mathrm{~m}$ ü. M.) stellte ich in der etwa $3 \mathrm{~m}$ mächtigen lehmig-sandigen Deckschicht ein «ziemliches Durcheinander» fest: Dicht neben $2 \mathrm{~m}$ mächtigen karbonatfreien Partien konnten Stellen mit beträchtlichem Karbonatgehalt gefunden werden. Dieser kann z. T. auf kleine Karbonatkonkretionen zurückgeführt werden, die wahllos in der ganzen Tiefe des Aufschlusses verteilt sind. Ebenfalls unregelmäßig verteilt finden sich in verschiedenen Tiefen Gerölle bis zu $6 \mathrm{~cm}$ Durchmesser. Ein in $90 \mathrm{~cm}$ Tiefe gefundener Topfscherben wurde von Prof. Dr. W. Guyan (mdl. Mitt.) als eindeutig mittelalterlich interpretiert! Die Regellosigkeit in der Deckschicht und der Fund dieses Scherbens deuten auf Hochflutablagerungen hin, besonders da die Oberfläche dieses Aufschlusses nur 1,5 bis $2 \mathrm{~m}$ höher als der $200 \mathrm{~m}$ davon entfernte Halbbach liegt.

Diese beiden Aufschlüsse zeigen deutlich, daß von einer Lößdecke, wie sie bisher angenommen wurde 
(vgl. Hantke, 1967), nicht die Rede sein kann, wenn auch nicht auszuschließen ist, $\mathrm{da} ß$ an anderer Stelle im Klettgau tatsächlich echter Löß zu finden ist.

\subsection{Grube Nr. 1 (Hungerbuck)}

Der Aufschluß, dessen Lage in Fig. 2 gut zu erkennen ist, liegt bei Pt. 678 120/282 000; 432 m ü. M. Der Hungerbuck bildet eine Art «Vorhügel» zum Hasenberg. Er wurde in der geologischen Karte (Hantke, 1967) als Drumlin bezeichnet, was vom morphographischen Standpunkt her vertretbar ist. An der Aufschlußwand (vgl. Fig. 3), die in NordSüd-Richtung verläuft, wird sofort klar, daß es sich bei der obersten Schicht nicht um normalen Löß handelt, denn es konnten darin gut gerundete Gerölle verschiedenster Größe gefunden werden, darunter auch ein Amphibolit von $18 \mathrm{~cm}$ Durchmesser in $1 \mathrm{~m}$ Tiefe. Karbonatgesteine konnten bis jetzt keine gefunden werden. Diese "obere Deckschicht» von maximal $2 \mathrm{~m}$ Mächtigkeit ist vollkommen karbonatfrei. Hofmann und Peters (1969, p. 12) deuteten sie als «... vermutlich sekundär entkalkten, rißeiszeitlichen Schwemmlehm». Im untern Teil dieser Schicht (Probe e in Fig. 3) kann das Feinmaterial von der Korngröße her als Löß bezeichnet werden, im obern Teil scheint das Material - der Fingerprobe nach zu schließen - bedeutend toniger zu werden; Korngrößenanalysen stehen jedoch noch aus.

Darunter folgt die maximal $80 \mathrm{~cm}$ mächtige, karbonathaltige «untere Deckschicht», die typischen Lößcharakter aufweist, ohne Grobmaterial (größer als $2 \mathrm{~mm}$ ).
$\mathrm{Da}$ es sich in diesen obersten $3 \mathrm{~m}$ tatsächlich um eine echte Zweischichtigkeit handelt, wird neben dem Unterschied im Grobmaterial dadurch belegt, daß der Karbonatgehalt im Grenzbereich dieser beiden Schichten nicht allmählich, sondern fast schlagartig von 0 auf $26 \%$ ansteigt. Erste Untersuchungen lassen den Schluß zu, daß es sich bei diesem Karbonat mindestens teilweise um sekundäre Ausscheidungen handelt. Das könnte bedeuten, daß dieser Löß den $\mathrm{C}_{\mathrm{c}}$-Horizont (= Kalkanreicherungshorizont) eines fossilen Restbodens darstellt. Das Liegende dieser Deckschichten bildet - wiederum scharf gegen diese abgesetzt - kalkhaltiger Sand, der von Schräg- und Kreuzschichtungen sowie einzelnen Geröllschnüren durchsetzt ist (Abb. 2). Nach den Angaben eines Arbeiters, der mit dem Auffüllen dieser im Sommer 1972 noch rund $5 \mathrm{~m}$ in den Sand reichenden Grube beschäftigt war, sollen diese sandigen Ablagerungen sicher eine Mächtigkeit von $20 \mathrm{~m}$ aufweisen. Sie dürften unzweifelhaft rißeiszeitlichen Alters sein.

Die schon erwähnte scharfe Grenze des Sandes gegen die untere Deckschicht wird zusätzlich durch einen kleingewellten, weißen, fast reinen Karbonathorizont von 1 bis $2 \mathrm{~cm}$ Mächtigkeit betont $(95 \%$ $\mathrm{CaCO}_{3}$ ). Dieser Horizont liegt eindeutig zwischen Sand und Löß (vgl. Abb. 3) und dürfte somit kaum als $\mathrm{C}_{\mathrm{c}}$-Horizont eines Bodens $\mathrm{zu}$ interpretieren sein; ganz auszuschließen ist allerdings diese Möglichkeit nicht. Es besteht aber die Vermutung einer großräumigen Verbreitung dieses Horizontes, denn beim Bau der Grundwasserfassung Oberhallau (EAWAG, 1969) wurde in rund einem Meter Tiefe eine (allerdings $80 \mathrm{~cm}$ mächtige) Seekreide erbohrt. Diese Seekreide liegt dort zwischen 429,4 m und $430,2 \mathrm{~m}$ ü. M. Diese verblüffend gleiche Höhenlage

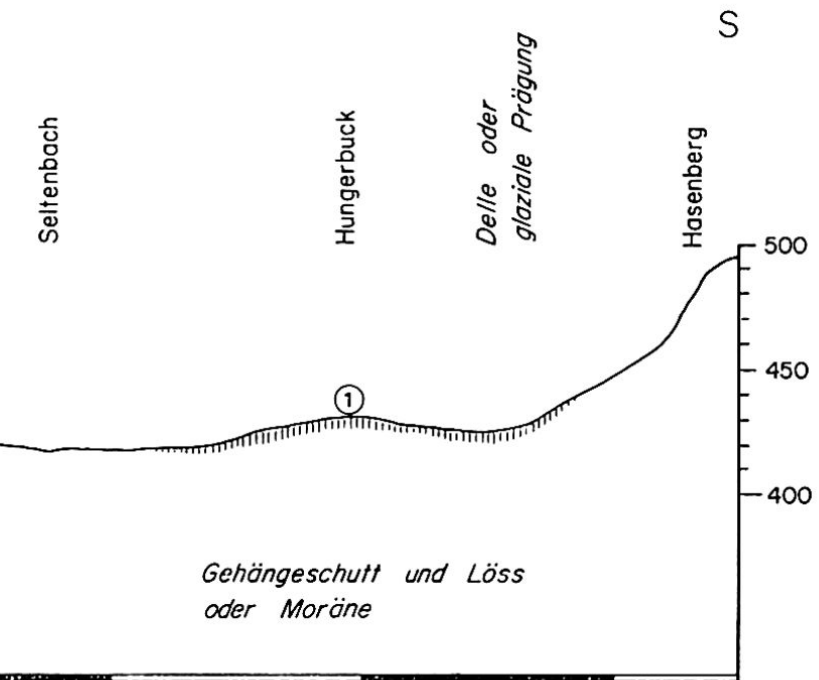

Figur 2. Profil durch das Klettgau. Die in Fig. 1 mit «Löß» bezeichneten Flächen sind senkrecht schraffiert dargestellt, die kursive Schrift bezieht sich auf die in dieser Arbeit erwähnten Interpretationsmöglichkeiten dieser Gebiete 


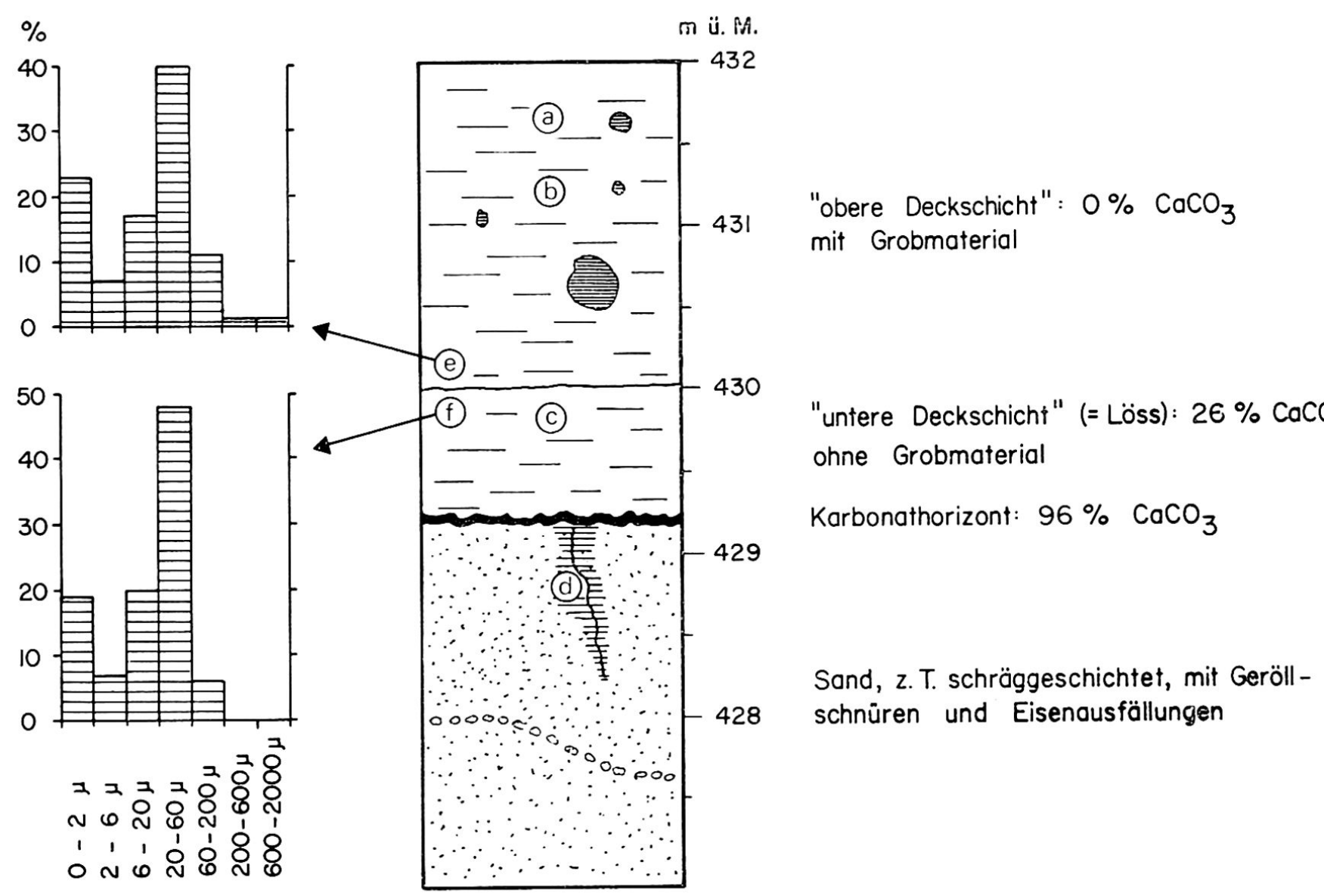

Figur 3. Schematische Darstellung des Aufschlusses auf dem Hungerbuck. An den Stellen a bis $f$ wurden die Proben für die Analysen entnommen (d bezieht sich nur auf die sekundären Eisenausscheidungen im Sand). Links die Korngrößenanalysen der Proben e und $f$

der Karbonatschichten in Oberhallau und am Hungerbuck kann allerdings rein zufälliger Natur sein.

Erwähnenswert im Sand sind ferner mehrere durch Eisen braunrot gefärbte Stellen (vgl. Abb. 3): Eisenausscheidungen umgeben mit Lehm gefüllte, dünne Spalten, die sich von der Untergrenze des Karbonathorizontes 0,5 bis $2 \mathrm{~m}$ in den Sand hinunterziehen. Diese Spalten stellen vermutlich Leitbahnen dar, in denen tonhaltige Lösungen mit einem gewissen Eisengehalt nach unten zirkulierten und dabei auch den umgebenden Sand durchtränkten.

Bevor wir uns den Deutungsmöglichkeiten dieses Aufschlusses zuwenden, seien noch einige Analysen erwähnt (Lage der Proben in Fig. 3 eingezeichnet):

$\begin{array}{ccccc}\text { Probe } & \begin{array}{c}\text { Gehalt an } \\ \text { org. C }\end{array} & \mathrm{Fe}_{\mathrm{d}}{ }^{*} & \mathrm{Fe}_{\mathrm{o}}{ }^{*} & \mathrm{Fe}_{\mathrm{o}} / \mathrm{Fe}_{\mathrm{d}}{ }^{*} \\ \text { a } & 0,7 \% & 15,8 \% 0 & 2,0 \% 0 & 0,13 \\ \text { b } & 0,2 \% & 18,6 \% 0 & 2,2 \% 0 & 0,12 \\ \text { c } & 0,1 \% & 10,9 \% 0 & 0,8 \% 0 & 0,07 \\ \text { d } & - & 12,4 \% 0 & 1,6 \% 0 & 0,13\end{array}$

* Sinn und Methodik dieser Analysen sind im Anhang beschrieben.

Wenn man von allen im Anhang erwähnten Unsicherheitsfaktoren absieht, liegt bei unserem Pro- fil die Vermutung nahe, daß die Eisenausscheidungen im Sand altersmäßig mit der oberen Deckschicht zusammenhängen, denn die Proben a, b und d zeigen praktisch den gleichen Aktivitätsgrad. Außerdem ist wahrscheinlich, daß zwischen unterer und oberer Deckschicht altersmäßig ein gewisser Unterschied besteht, was für die Genese des Hungerbucks von Bedeutung ist.

\section{Gegenüberstellung von zwei Deutungsmöglichkeiten}

Nach A. Pencks Meinung (1901) reichte der Würmgletscher bei seiner maximalen Ausdehnung bis zum «Lusbüel» (Pt. 685 600/282 800), also etwa $2 \mathrm{~km}$ ins Klettgau hinein. Nach Huber (1956) und Graul (1962) sollte das Würmeis nicht einmal so weit geflossen sein, der Maximalstand ist nach diesen beiden Autoren in der Gegend der heutigen "Engi» zu suchen. Diese Ansichten zeigen eindeutig, daß für unser Untersuchungsgebiet während der Würmkaltzeit eine glaziale Úberprägung ausgeschlossen werden kann, was im Hinblick auf die Genese des Hungerbucks wichtig ist. Etwas abweichend vom ursprünglichen Gedanken über Lößuntersuchungen seien nun zwei genetische Deutungsmöglich- 


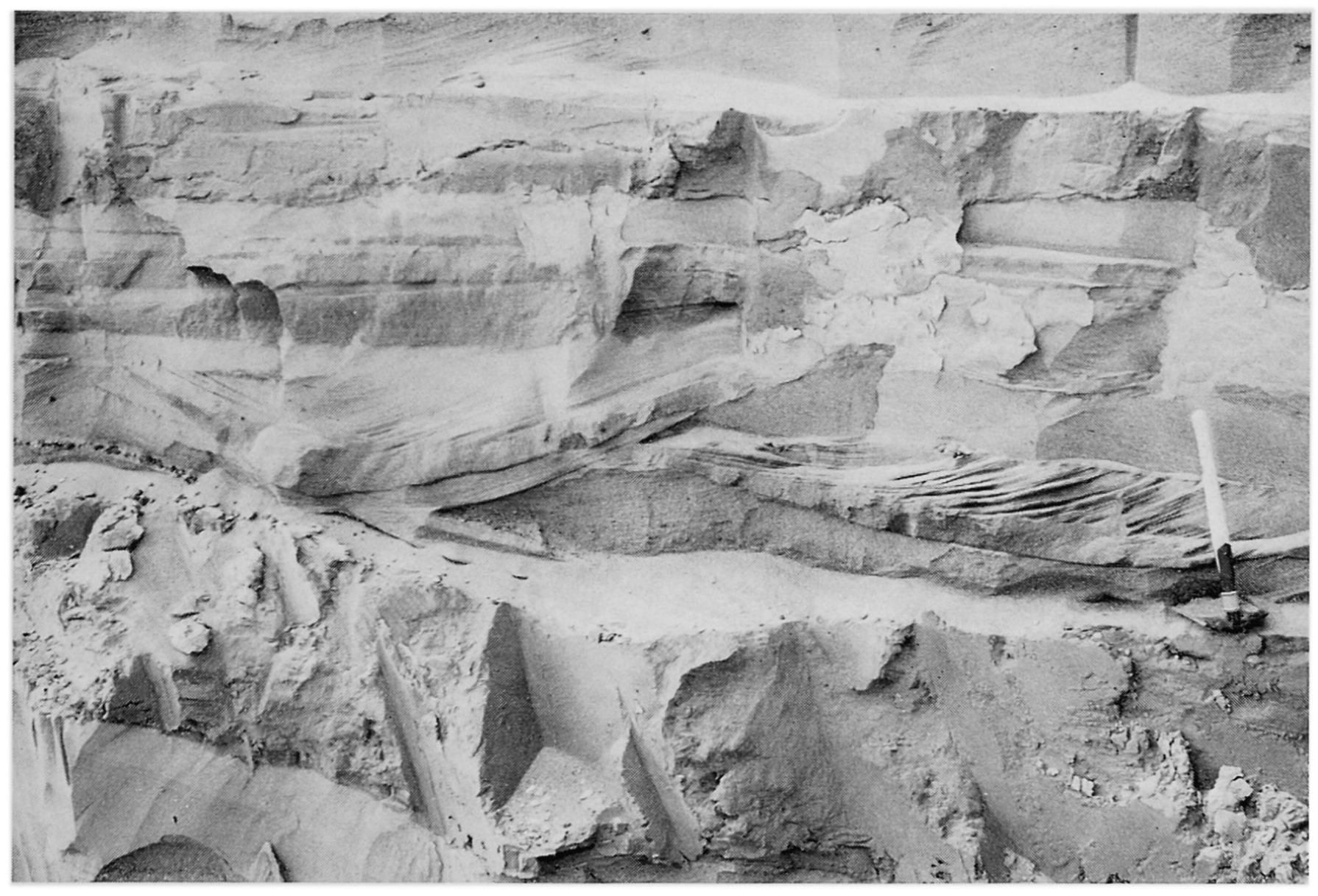

Abbildung 2. Ausschnitt aus den Sandschichten am Hungerbuck. Gut sind die vom Wind herauspräparierten Schrägschichtungen (beim Spaten) sowie eine beginnende Geröllschnur (in der Mitte des linken Bildrandes) zu erkennen

keiten des Hungerbucks und seines benachbarten Geländes dargelegt.

In jedem Fall aber schwierig zu deuten ist der Karbonathorizont. Der erste Hinweis einer möglichen Entstehung fand sich bei Rohdenburg (1965), der von einem weißen «Lockerdauch» (Süßwasserkalk) spricht, welcher in einem dünnen Bändchen abgelagert worden ist (p. 38). An anderer Stelle (p. 40) erwähnt er einen seekreideartigen Lockerdauch. Handelt es sich aber bei dieser welligen Struktur des Karbonathorizontes um eine natürliche Ablagerungsform, oder ist sie erst nachträglich (evtl. unter Einfluß des Permafrostes) entstanden?

\subsection{Erste Deutungsmöglichkeit}

Es handelt sich beim Hungerbuck - wie aus der geologischen Karte (Hantke, 1967) ersichtlich ist um einen rißzeitlichen Drumlin. Sand und Löß im Aufschluß repräsentieren einen (vielleicht nur lokalen) eisfreien Abschnitt. Die obere Deckschicht müßte dann als Moräne eines vorstoßenden Rißgletschers aufzufassen sein, der diesen Drumlin bil- dete. Die morphologische Tätigkeit während der Würmkaltzeit bliebe damit auf die Denudation beschränkt, deren Ausmaß heute nicht mehr bestimmt werden kann. $\mathrm{Zu}$ dieser Theorie gibt es aber verschiedene Ungereimtheiten:

- Die Untergrenze dieser «Moräne» fällt nach den bisherigen groben Messungen im ganzen Aufschluß mit einer Neigung von $2-3^{\circ}$ gegen das Haupttal hin ein. Die Karbonatschicht liegt - nebenbei bemerkt - praktisch horizontal.

- Da die Rißmoräne aller Wahrscheinlichkeit nach ursprünglich kalkhaltig gewesen ist, kann die scharfe Entkarbonatisierungsgrenze zum Löß hin nur schwierig erklärt werden.

- Warum hat ein Gletscher, der eine ursprünglich sicher mehr als $2 \mathrm{~m}$ mächtige Moräne ablagerte, diesen aus ganz lockerem Sand aufgebauten Hügel nicht einfach wegerodiert (Permafrost?)?

\subsection{Zweite Deutungsmöglichkeit}

Aus verschiedenen Beobachtungen kann man sich die obere Deckschicht als einen Solifluktionsschutt 
aus (mit Löß vermischtem) Moränenmaterial vorstellen, der vom Hasenberg heruntergerutscht ist. Das den Hungerbuck vom Hasenberg trennende Tal muß später, als Delle oder Dellental (vgl. weiter unten), entstanden sein. Das Alter der oberen Deckschicht ist noch ungeklärt; sowohl Riß als auch Frühwürm kommen in Frage. Im ersten Fall (rißzeitliches Alter) hätte sich sowohl der Eem-Boden als auch der holozäne Boden in der oberen Deckschicht entwickelt. Im zweiten Fall (frühwürmzeitliches Alter) müßte sich der Eem-Boden auf dem damals viel mächtigeren Löß ausgebildet haben; er ist im Jungwürm durch den ihn überwandernden Solifluktionsschutt (obere Deckschicht) bis auf den Karbonatanreicherungshorizont (untere Deckschicht) abgetragen worden. Nicht ganz in dieses Bild paßt aber das Aussehen der Delle (Abb. 4): Mit einer Länge von einem Kilometer, einer maximalen Breite von etwa $350 \mathrm{~m}$ (beim Hungerbuck) und einem Gefälle von knapp $1^{\circ}$ scheint sie eine etwas eigenartige Stellung in der von Semmel (1968, p. 102 f.) erwähnten morphographischen Gliederung einzunehmen, in der er von "Dellen» spricht, wenn die Neigung mehr als $3^{\circ}$ und die Länge weniger als $1 \mathrm{~km}$ beträgt, von «Dellentälern" hingegen bei geringerer Neigung und einer Länge von mehreren Kilometern. Auch die rekonstruierte Schuttmächtigkeit (angenommen wurde ein $\mathrm{Ge}$ fälle der Deckschicht von $2^{\circ}$ ) im heutigen Dellentiefsten müßte mindestens $15-20 \mathrm{~m}$ betragen haben, was einem erstaunlich großen Abtrag während der Würmkaltzeit oder eines Teils derselben gleichkommt. Nur im Falle eines rißzeitlichen Alters der oberen Deckschicht könnte man sich vielleicht eine spätere (aber immer noch rißzeitliche) Vorprägung der Delle durch Eis oder Schmelzwasser vorstellen.

\section{Schlußfolgerungen}

Versuche, aus dem Klettgauer «Löß» den chronologischen Ablauf wenigstens eines Teils der Würmkaltzeit zu rekonstruieren, konnten nicht angestellt werden, da offensichtlich in diesem Gebiet der Löß nicht so weit verbreitet ist, wie bisher angenommen wurde. In der schwach welligen Ebene des Haupttales muß vermehrt mit holozänen Hochflutablagerungen gerechnet werden; theoretisch sollte aber doch in den etwas höher liegenden Gebieten Löß anzutreffen sein.

Was die Genese der hangnahen Zone anbelangt, so kann erst dann volle Klarheit geschaffen werden, wenn die zeitliche Einstufung der einzelnen Schichten geklärt ist. Ziemlich sicher ist aber, daß in diesen Gebieten der Abtrag vorherrschte; es sind hier also kaum größere Lößvorkommen zu erwarten.

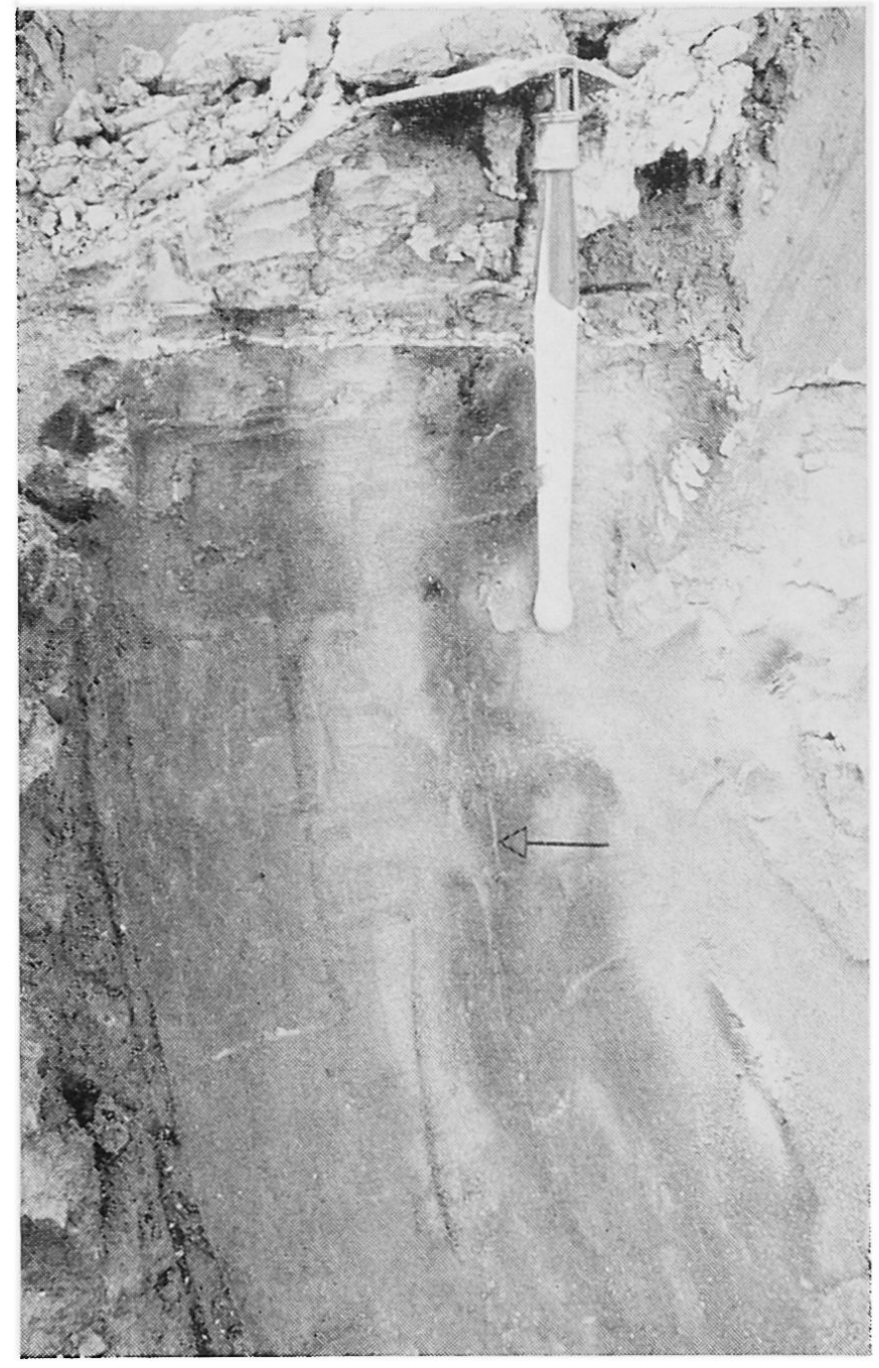

Abbildung 3. Ausschnitt aus dem Aufschluß am Hungerbuck. Im oberen Teil des Bildes die untere Deckschicht, in der Mitte des Spatenstiels der Karbonathorizont, darunter Sand (hell) mit Eisenausscheidungen (dunkel). Deutlich ist bei der Ausscheidung rechts im Bild die verlehmte Spalte zu sehen (Pfeil)

\section{Anhang: Bestimmung der pedogenen Eisenoxide}

Für die genaue Durchführung der Versuche sei auf Schwertmann (1964) verwiesen. Bei den pedogenen Eisenoxiden (bzw. -Hydroxiden und -Oxidhydraten) handelt es sich um Eisenverbindungen, die durch die Verwitterung aus den eisenhaltigen Mineralien (= pyrogene Eisenverbindungen) freigesetzt wurden. Das freigesetzte Eisen liegt zuerst in röntgenamorpher (= nichtkristalliner) Form vor. Mit saurer Oxalatlösung ist es im Dunkeln möglich, nur diese Eisenverbindungen zu extrahieren (= $\mathrm{Fe}_{0}$ ).

Im Boden wandeln sich die amorphen Eisenverbindungen im Verlauf der Zeit zu kristallinen Eisenverbindungen (in erster Linie Goethit) um. Mit der Dithionit-Citrat-Extraktion können zusätzlich zum $\mathrm{Fe}_{0}$ auch diese kristallinen Eisenverbindungen er- 


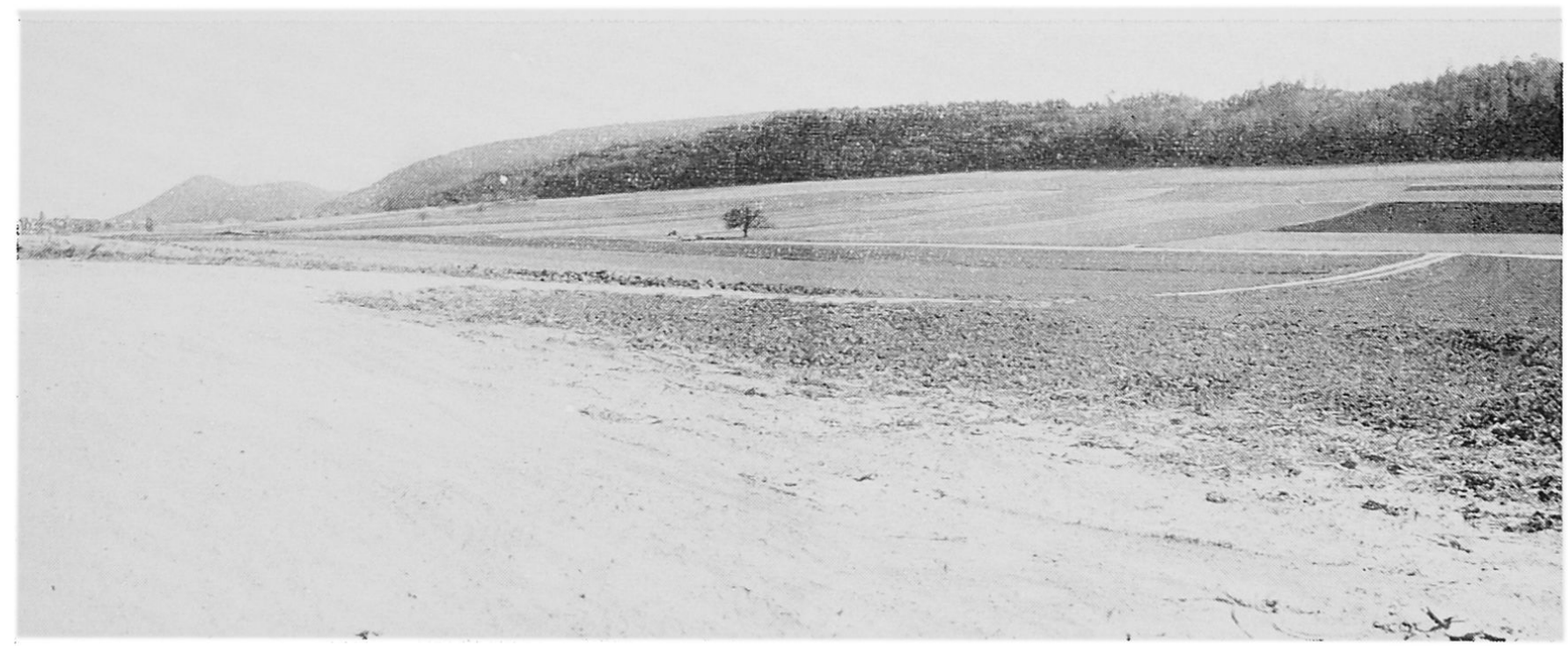

Abbildung 4. Blick vom Hungerbuck über die «Delle» gegen den Hasenberg. Die fast nur dank der Wege erkennbare Delle ist von links nach rechts geneigt. Die Distanz zum Wald ganz rechts beträgt etwa $500 \mathrm{~m}$

faßt werden $\left(=\mathrm{Fe}_{\mathrm{d}}\right)$. Generell kann gesagt werden, $\mathrm{da} \beta$ der Anteil von $\mathrm{Fe}_{0}$ am gesamten pedogenen Eisen mit zunehmendem Alter eines Bodens abnimmt: Das Verhältnis $\mathrm{Fe}_{0} / \mathrm{Fe}_{\mathrm{d}}$, der sogenannte Aktivitätsgrad der Eisenoxide, wird also mit zunehmendem Alter immer kleiner.

Bei der Interpretation muß allerdings berücksichtigt werden, daß eine Umwandlung von $\mathrm{Fe}_{\mathrm{o}}$ zu $\mathrm{Fe}_{\mathrm{d}}$ durch Staunässe oder organische Verbindungen verzögert werden kann. Bei Vergleichen sollten auch die Bodenhorizonte einander entsprechen (vgl. Schlichting und Blume, 1962).

\section{Literaturverzeichnis}

EAWAG: Die Grundwasserverhältnisse des Klettgau. Geotechn. Büro Dr. A. von Moos, 1969.

Graul H.: Geomorphologische Studien zum Jungquartär des nördlichen Alpenvorlandes, Teil I. Heidelberger Geogr. Arbeiten, 1962.

Hantke R.: Geologische Karte des Kantons Zürich und seiner Nachbargebiete. Vierteljahresschrift der naturf. Ges. in Zürich, Jg. 112, H. 2, 1967.

Hofmann F. und Peters T.: Untersuchungen über die Verwendbarkeit schweizerischer Rohstoffe als Bindetone für Gießereiformsande. Beitr. zur Geologie der Schweiz, Geotechn. Serie, Lieferung 47, 1969.
Huber R.: Ablagerungen aus der Würmeiszeit im Rheintal zwischen Bodensee und Aare. Vierteljahresschrift der naturf. Ges. in Zürich, Jg. 101, Abh. $1,1956$.

Penck A.: Die Glacialbildungen um Schaffhausen. Sep. aus: Denkschriften der Schweiz. Naturf. Ges., Bd. XXXV, 1901.

Rohdenburg H.: Untersuchungen zur pleistozänen Formung am Beispiel der Westabdachung des Göttinger Waldes. Gießener Geographische Schriften, H. 7, 1965.

Schlichting E. und Blume H.-P.: Art und Ausmaß der Veränderungen des Bestandes mobiler Oxide in Böden aus jungpleistozänem Geschiebemergel und ihren Horizonten. Zschr. für Pflanzenernährung, Düngung, Bodenkunde, Bd. 96, H. 2, 1962.

Schwertmann U.: Differenzierung der Eisenoxyde des Bodens durch Extraktion mit Ammoniumoxalaî-Lösung. Zschr. für Pflanzenernährung, Düngung, Bodenkunde, Bd. 105, H. 3, 1964.

Semmel A.: Studien über den Verlauf jungpleistozäner Formung in Hessen. Frankfurter Geogr. Hefte, 45, 1968.
Adresse des Verfassers:
Dr. Peter Fitze
Geographisches Institut der Universität Zürich
Blümlisalpstraße 10, 8006 Zürich 